\title{
Notes sur le rostre des siliques de Brassica oleracea.
}

\author{
Par \\ J. GOLIŃSKA.
}

Le fruit de Crucifères, qui se forme de deux ou, comme affirme Saunders (7), de quatre carpelles, consiste d'une partie valvaire et du rostre. Les dimensions de ces deux parties varient beaucoup; chez les choux la silique a de 6 à $9 \mathrm{~cm}$ de longueur et le bec n'a que 4 à $6 \mathrm{~mm}$, rarement il atteint $15 \mathrm{~mm}\left(\mathrm{Schulz}^{8}\right)$. La cloison (septa) de la partie valvaire se prolonge dans le rostre, qui est rempli de mésocarpe spongieux. Il contient souvent de 1 à 5 graines, particularité qui parmi toutes les Crucifères ne se trouve que chez les Brassicinae (Schulz 8 p. 11).

L'observation, que j'ai faite sur plusieurs plantes à graines des choux pommés d'une lignée selectionnée des choux var. „de Varsovie“, a montré que le pour-cent des rostres contenant des graines varie beaucoup chez les différentes plantes. J'ai trouvé des plantes dont tous les rostres étaient vides et d'autres qui avaient de 70 à $89 \%$ becs à graines. Les mesurages, exécutés sur quelques centaines de siliques, prouvent que la longueur du rostre n'est pas trop étroitement liée avec la longueur du fruit (cfr. la table I); une corrélation plus prononcée a été trouvée entre la longueur du rostre et la quantité de graines qu'il contenait.

Ces faits, obtenus en 1925, donnèrent l'idée d'étudier plus soigneusement les causes qui influencent le développement des rostres et de comparer les qualités des graines provenues des rostres et des parties valvaires des siliques. Dans ce but, on sema en 1926 les graines, prises de 4 plantes de var. „de Varsovie“, qu'on avait observées l'année précédente; deux plantes, ayant plus de $50 \%$ de rostres fertiles et deux autres aux rostres vides. De plus on sema séparément 


\section{Table I.}

Coefficients de corrélation.

\begin{tabular}{|l|c|c|}
\hline $\begin{array}{c}\text { Numéros } \\
\text { des } \\
\text { plantes }\end{array}$ & $r_{1}=\frac{\text { longueur de silique }}{\text { longueur de rostre }}$ & $r_{2}=\frac{\text { longueur de rostre }}{\text { nombre de graines dans le rostre }}$ \\
\hline $1 / 41$ & +0.220 & +0.403 \\
$1 / 39$ & +0.432 & $\left.-{ }^{2}\right)$ \\
$6 / 12$ & +0.322 & $\left.-{ }^{2}\right)$ \\
$6 / 24$ & +0.571 & +0.607 \\
$\left.6 / 24-29^{1}\right)$ & +0.452 & +0.531 \\
\hline
\end{tabular}

les graines tirées des becs d'une de ces plantes. En automne, 20 pieds de chaque catégorie de choux furent serrés dans la cave pour les faire grainer en 1927.

La récolte d'automne prouva que les pieds issus des graines de la partie valvaire avaient des pommes plus lourdes et mieux formées que ceux des graines de rostre: les premiers pesaient en moyenne 1387 gr. et les autres 973 gr. Ce résultat est facile à expliquer, si l'on compare le poids de cent graines provenant des deux parties du fruit; à savoir:

100 graines du rostre 100 graines de la partie valvaire
plante $\mathrm{Nr} 16$
$0.348 \mathrm{gr}$
$0.422 \mathrm{gr}$
plante $\mathrm{Nr} 63$
$0.363 \mathrm{gr}$
$0.545 \mathrm{gr}$

Il faut noter que la plupart des graines tirées du rostre étaient ridées, mais presque de la même grandeur que les graines de la partie valvaire. Ainsi le poids inférieur des plantes issues des graines du rostre est dû au mauvais développement de ces graines et non à leur dimension, ce qui s'accorde avec les recherches de Kotowski (5)

Les plantes récoltées en 1926 et qui grainaient en 1927, devaient servir à résoudre la question, à quel point la fertilité des rostres peut être influencée par la différente alimentation des jeunes siliques. On profita des différences, qui existent à cet égard entre la tige principale et les tiges latérales d'une plante ( $\mathrm{G}$ o e bel 1 et 2 ), et on tailla les plantes à graines de manière suivante:

1. Plantes à tige principale, dont toutes les tiges latérales furent coupées.

2. Plantes aux tiges latérales, tige principale coupée.

1) Plante à graines de 1927; les autres grainées en 1925 .

2) Plantes $\mathrm{Nr} 1 / 39$ et $6 / 12$ avaient des rostres stériles. 
Chacune de ces deux séries contenait des plantes, auxquelles les sommets de tous les rameaux furent pincés le 10 juin, c'est à dire à l'époque où la majorité des plantes était en pleine floraison et que les premières siliques furent nouées. Toutes les fleurs formées après cette date furent détruites (plantes groupe $A$ ). Les plantes du groupe $B$ ne furent pas pincées et florissaient sans entraves. Il y avait aussi des plantes témoins, qui n'étaient ni taillées, ni pincées, mais il n'en resta que 4 jusqu'à la récolte.

La table II présente les données concernant les plantes à graines de 1927. Il en résulte que les siliques des choux pincés sont plus lourdes que les autres et que, si l'on peut se permettre de tirer une conclusion des données concernant 8 plantes, les fruits attachés aux tiges principales ont des conditions du développement plus favorables que les fruits des branches latérales.

Mais ni la taille, ni le pincement des porte-graines des choux n'exercent nulle influence sur la fertilité des rostres: dans tous les groupes de ces plantes le pour-cent des rostres à graines varie largement; à savoir:

$$
\begin{aligned}
& \text { Série } 1 \text { gr. } B-\text { de } 5 \% \text { - à } 57 \% ; \\
& \text { Série } 1 \text { gr. } A-\text { de } 0 \% \text { - à } 41.5 \% ; \\
& \text { Série } 2 \text { gr. } B-\text { de } 0 \%-\text { à } 59 \% ; \\
& \text { Série } 2 \text { gr. } A-\text { de } 0.5 \%-\text { à } 61.5 \% \text {; } \\
& \text { Plantes témoins - de } 0 \% \text { - } 53 \% \text { (cfr. la table II). }
\end{aligned}
$$

Le plus haut pour-cent des rostres fertiles donnèrent les descendants du pied $6 / 24$, qui avait en $1925-70 \%$ des becs à graines, sans différence si les plantes à graines provenaient des semences tirées des becs ou des parties valvaires (cfr. tab. II). Ces données font admettre que la fertilité du rostre est un caractère héréditaire et dépend des facteurs génétiques. Korpechenko (4) trouva même que chez les hybrides Raphanus sativus L. $\times$ Brassica oleracea L., dont les fruits étaient à moitié des siliques des choux, à moitié des fruits des radis (fig. $3 A_{1}, A_{2}$ ), „les particularités morphologiques des hybrides se trouvent en rélation absolue avec leurs complets des chromosomes“ (4, p. 345); et "qu'avec le croíssement du pour-cent des chromosomes des choux grandit aussi, chez ces plantes, la longueur de la partie valvaire du pistil“ (4 p. 344).

Revenant à la table II, nous voyons que, chez les descendants des plantes aux becs stériles (plantes $1 / 13$ et $1 / 42$ ), le pour-cent des rostres à graines varie de $0 \%$ à $47 \%$ et en moyenne ils ont $19 \%$ et $13 \%$ des rostres fertiles. Un pareil pour-cent moyen $(15 \%)$ des 
becs à graines donnent les porte-graines, dont la plante mère avait en 1925 plus de $50 \%$ des rostres à graines.

Or, si la fertilité du rostre est un caractère héreditaire, les portegraines observés ne sont point une race pure, quoiqu'ils descendent d'une lignée sélectionnée. Ils montrent beaucoup de particularité dans les différents ćaractères, qu'on ne peut pas considérer comme résultant de la taille ou du pincement; voici quelques exemples. Le poids moyen d'une silique varie chez les plantes qui n'etaient

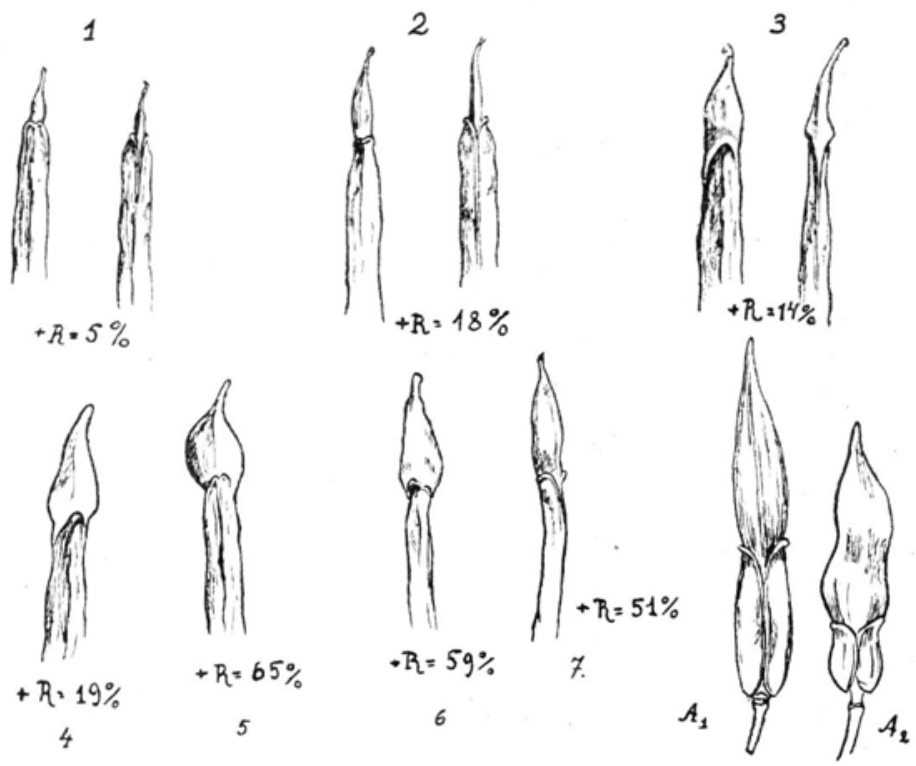

Fig. 3. Dessins $1^{0}-7^{0}$. Moitiés supérieures des siliques des diverses plantes, ayant le pourcent de rostres à graines comme il suit: $1 \%-5 \%, 2 \%-18 \%, 3 \%-14 \%$, $4 \%-19 \%, 5^{0}-65 \%, 6^{0}-59 \%, 7^{0}-51 \%$. Toutes les figures sont à la même échelle. Dessins $A_{1}-A_{2}$ d'après $\mathrm{K}$ arpe che n ko (4) deux fruits des hybrides Raphanus sativus L. $\times$ Brassica oleracea L.; $A_{1}$ - un hybride pentaploide, qui avait 18 chromosomes de Raph. sat. et 18 de Brassica oleracea. $A_{2}-$ un hybride tetraploide au 18 chromosomes de Raph. sat. et 9 de Brassica oleracea.

pas pincées de $0.096 \mathrm{gr}$ à $0.315 \mathrm{gr}$ et de $0.192 \mathrm{gr}$ à $0.410 \mathrm{gr}$ chez les plantes pincées. Le rapport longueur de silique trouvé pour divers porte-graines à tige principale est:

$\frac{7 \mathrm{~cm}}{1 \mathrm{~cm}}=7 ; \frac{8.7 \mathrm{~cm}}{0.8 \mathrm{~cm}}=11 ; \frac{8.3 \mathrm{~cm}}{1.8 \mathrm{~cm}}=4.6$ pour plantes qui ne furent pas pincées,

$\frac{11 \cdot 9 \mathrm{~cm}}{1 \cdot 7 \mathrm{~cm}}=7 ; \frac{8 \cdot 7 \mathrm{~cm}}{0 \cdot 6 \mathrm{~cm}}=14 ; \frac{9 \cdot 8 \mathrm{~cm}}{1 \cdot 9 \mathrm{~cm}}=5 \cdot 3$ pour les plantes pincées. 


\begin{tabular}{|c|c|c|c|c|c|c|}
\hline \multirow{4}{*}{$\begin{array}{c}n \\
0 \\
0 \\
0 \\
0 \\
0 \\
0 \\
0 \\
0 \\
0 \\
+ \\
\frac{\pi}{a} \\
\frac{\pi}{a}\end{array}$} & \multirow{2}{*}{ 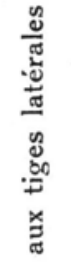 } & 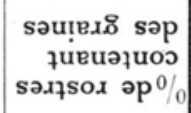 & \multicolumn{2}{|l|}{$n N \stackrel{n}{\sim} \frac{n}{m} \underset{\tilde{m}}{n}$} & $\dot{0} \approx n \approx$ & \multirow{2}{*}{ 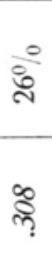 } \\
\hline & & 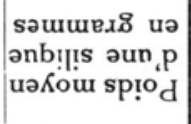 & 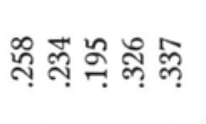 & ণิ & 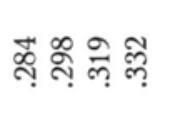 & \\
\hline & $\frac{0}{\frac{0}{0}}$ & 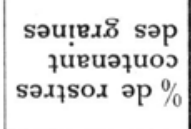 & $\stackrel{n}{\sigma}$ & ถุ่ & 0 & $\frac{8}{0}$ \\
\hline & $\stackrel{0}{.0}$ & $\begin{array}{l}\text { səuuuex. uə } \\
\text { әnb!!!s əun } 9 \\
\text { uəKou sp!od }\end{array}$ & ஸ̊ & ๓ి & กิ & ๙ุ \\
\hline in & है & 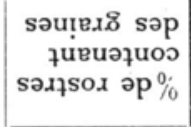 & $\circ \stackrel{n}{\sim}$ & $\stackrel{\theta}{\theta}$ & & \\
\hline $\begin{array}{l}0 \\
0 \\
0 \\
0 \\
0 .\end{array}$ & $\frac{\stackrel{n}{d}}{\frac{\pi}{2}}$ & 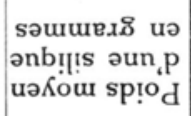 & $\stackrel{2}{\simeq}$ & $\frac{1}{7}$ & & \\
\hline $\begin{array}{r}0 \\
+ \\
0 \\
0\end{array}$ & 眎 & $\begin{array}{r}\text { səu!ex.8 səp } \\
\text { queuəquoว } \\
\text { səגі̧sox әр \% }\end{array}$ & $\stackrel{\infty}{\rightarrow}$ & $\frac{0}{8}$ & $9-+0 \cong$ & $\frac{e}{\pi}$ \\
\hline $\begin{array}{l}+\infty \\
= \\
= \\
=\end{array}$ & 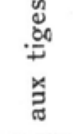 & 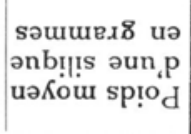 & 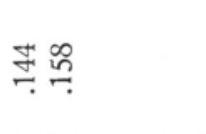 & $\stackrel{5}{?}$ & 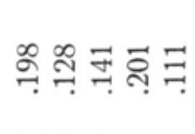 & $\stackrel{?}{?}$ \\
\hline $\begin{array}{l}n \\
0 \\
+\infty \\
0 \\
0\end{array}$ & $\frac{0}{\frac{2}{0}}$ & 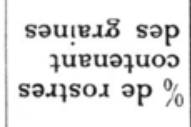 & $\sim \stackrel{n}{\sim} \infty \stackrel{n}{\sim}$ & $\frac{e}{6}$ & 0 으 n & 웃 \\
\hline a & $\underset{. \infty}{\infty}$ & 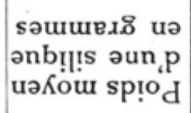 & 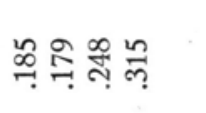 & ๗ิ & 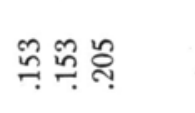 & 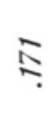 \\
\hline & 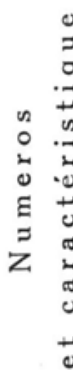 & 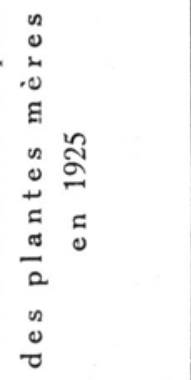 & 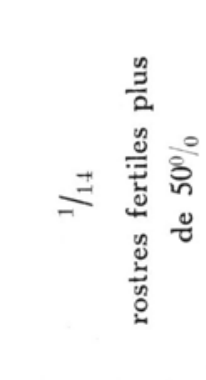 & 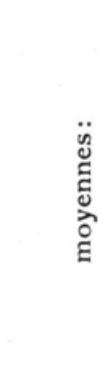 & 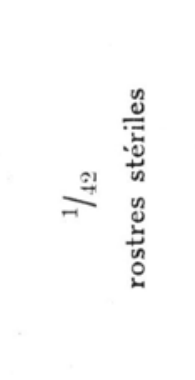 & 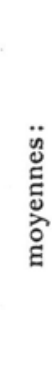 \\
\hline
\end{tabular}




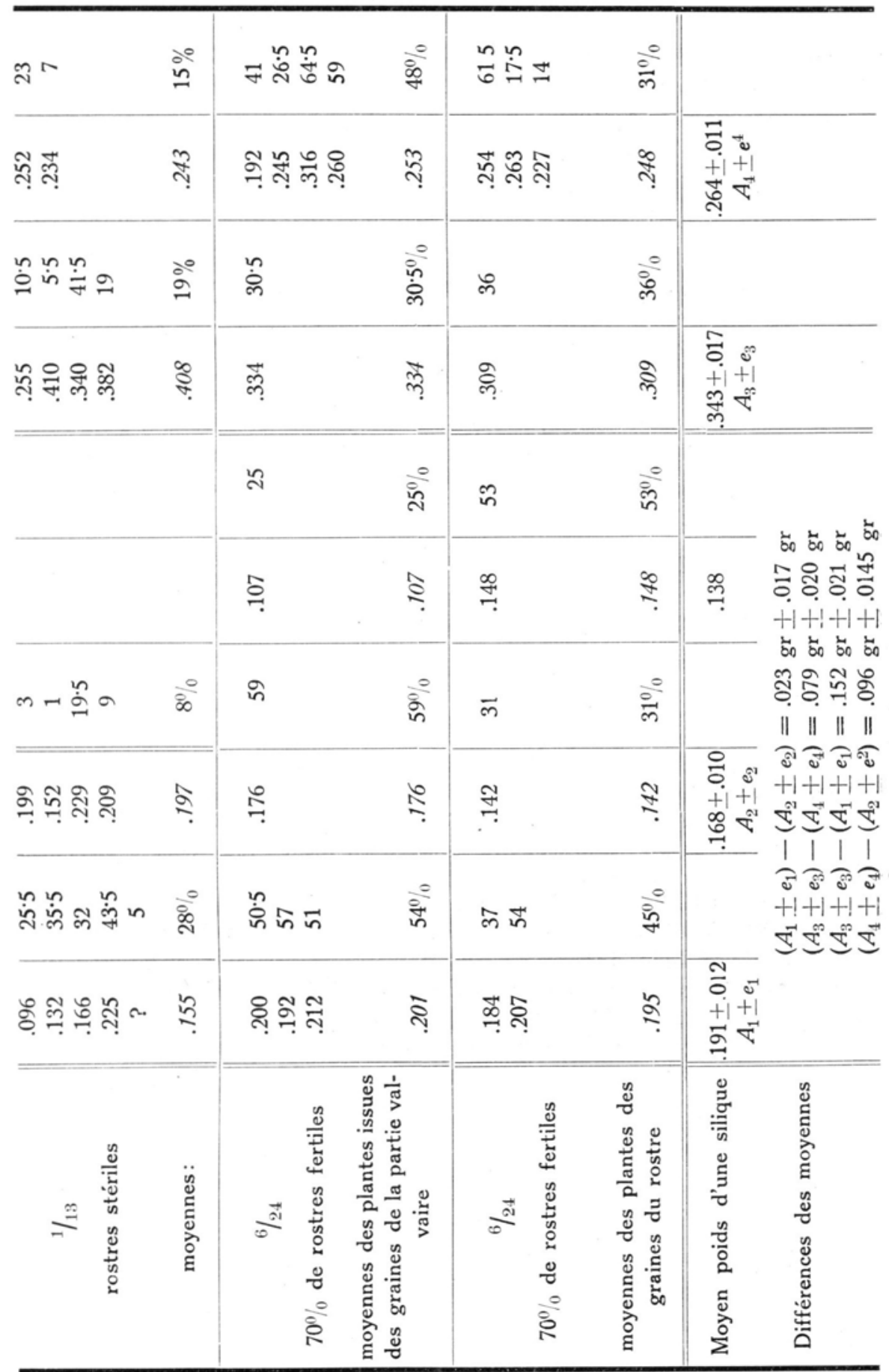


Pour juger de la différence de grandeur des semences récoltées des diverses plantes - voici l'oscillation du pour-cent des graines plus petites de diamètre que $1.5 \mathrm{~mm}$ dans le rendu d'une plante: plantes témoins - de $2 \cdot 6 \%$ à $82.3 \%$, plantes pincées à tige principale - de $3 \cdot 7 \%$ à $66 \%$. La fig. 3 montre les diverses formes du rostre trouvées parmi ces plantes à graines.

A mon grand regret, il me manque des données exactes, qui permettraient de juger d'autres caractères des porte-graines. Il me paraît, qu'en les étudiant plus attentivement, on parviendrait à connaître les différentes formes des choux homologues aux formes d'un nombre de Crucifères qui sont décrites par Sinskaia (6). L'étude des porte graines des Crucifères bisannuelles cultivés comme plantes potagères serait aussi d'importance pour leur sélection. Aujourd'hui on se borne à sélectionner les plantes de la première année, oubliant qu'on néglige ainsi une quantité de caractères de ces plantes, qui peuvent aider à établir des races pures.

Institut de Culture Potagère de l'École Supérieure d'Agriculture à Varsovie.

\section{Index bibliographique.}

1. Goebei, K. Einleitung in die Experimentelle Morphologie der Pflanzen. 1908.

2. G o e b e l, K. Organographie der Pflanzen. Bd. I. 1913.

3. $\mathrm{Heg}$ i, G. Illustrierte Flora der Mittel-Europa. Bd. IV, 1 Hälfte.

4. Korpe ch e n k o, G. D. Polyploid hybrids of Raphanus sativus $\mathrm{L} \times$ Brassica oleracea L. - Bull. of Applied Botany, Genetics and Plant-Breeding. Vol. 17. 1927.

5. Kotowski, F. Effect of Size of Seed on Plant Production. - Acta Soc. Bot. Poloniae III, Nr 2. 1925.

6. Sin s k a i a, E. A Contribution to the Knowledge of Regularity (parallelism) in the Variability of the Family Cruciferae. - Bull. of Appl. Botany and PlantBreeding. Vol. 13, 1922-1923.

7. S o unders, E. A reversionary character in the Stock (Matthiola incana). Annals of Botany. 37, 1923.

8. Schulz, O. Das Pflanzenreich. IV, 105. 